\title{
Note on the phase space of asymptotically flat gravity in Ashtekar-Barbero variables
}

\author{
Miguel Campiglia* \\ Raman Research Institute \\ Bangalore 560080, India and \\ Instituto de Física, Facultad de Ciencias \\ Montevideo 11400, Uruguay
}

\begin{abstract}
We describe the canonical phase space of asymptotically flat gravity in AshtekarBarbero variables. We show that the Gauss constraint multiplier must fall off slower than previously considered in order to recover ADM phase space. The generators of the asymptotic Poincare group are derived within the Ashtekar-Barbero phase space without reference to the ADM generators. The resulting expressions are shown to agree, modulo Gauss constraint terms, with those obtained from the ADM generators. A payoff of this procedure is a new expression for the generator of asymptotic rotations which is polynomial in the triad and hence better suited for quantum theory. Our treatment complements earlier description by Thiemann in the context of self-dual variables.
\end{abstract}

\section{INTRODUCTION}

In [1] Thiemann adapted the description of asymptotically flat canonical gravity in ADM variables [2] given in [3, 4] to Ashtekar variables [5, 6]. Among other things, he obtained the generators of the asymptotic Poincare group, showed their agreement with the ADM generators, and verified their Poisson brackets reproduce the Poincare algebra.

Here we revisit Thiemann's analysis in the context of real $S U(2)$ variables [7] with an arbitrary Barbero-Immirzi parameter $\gamma[8,9]$. We take the same parity conditions and leading fall-off terms as those used in [1, 3]. For subleading terms we use the more general fall-offs given in 4. We also show that, independently of the choice of subleading falloffs, the leading term of the Gauss constraint multiplier must fall off slower than what was considered in 1 if one wants to recover ADM phase space.

To obtain the Poincare generators, we follow the strategy of [3] where one seeks for boundary terms to the Hamiltonian and diffeomorphism constraint that yield well defined phase space functions when then lapse and shift have asymptotic values that correspond to Poincare transformations. This strategy is followed within the Ashtekar-Barbero phase space, without resorting to the ADM expressions.

For spacetime translations, we recover the known expressions given in [1, 6]. For boosts we obtain a generator, Eq. (3.46), that is shown to agree on the Gauss constraint surface with the one obtained in [1]. The situation is the most subtle with rotations. In [1] the generator

of rotations was obtained from the ADM generator. The resulting expression involves the

*Electronic address: campi@fisica.edu.uy 
spin connection and hence is non-polynomial in the triad. On the other hand the generator obtained here, Eq. (3.31), is polynomial in the triad. Showing that the two agree (modulo a Gauss constraint term with phase-space dependent multiplier) requires careful comparison of the expressions.

The motivation for the present study comes from its application to quantum theory. In particular, the expression for angular momentum obtained here facilitates the unitary implementation of asymptotic rotations described in [10].

The organization of the material is as follows. In section [I] we review the asymptotically flat ADM phase space as treated in [4. The section serves to set up notation, display the ADM Poincare generators for later comparison, and present the guiding principle of references [3, 4, that we follow in section III. In section III A we describe the Ashtekar-Barbero phase space counterpart of the ADM phase space of section III. In IIIB 1 we discuss the Gauss constraint and corresponding asymptotic behavior of its multiplier. In III B2 we discuss the Hamiltonian and diffeomorphism constraints, and in IIIC the Poincare generators. The discussion of rotations will be more detailed than for the other generators, since it is here that the comparison with ADM and Thiemann's expression is more subtle. In section IV] we show the Poincare generators of section III coincide with the ADM and Thiemann ones.

\section{REVIEW OF ADM CASE}

\section{Phase space}

In the asymptotically flat case, the Cauchy slice $\Sigma$ is such that it admits, outside a compact set, cartesian coordinates $x^{I}, I=1,2,3$ that extend to infinity. Let $\stackrel{\leftrightarrow}{q}_{a b}$ be the flat background metric associated with the cartesian coordinates so that $\stackrel{\circ}{q}_{I J}=\delta_{I J}$. Let $r:=\sqrt{\left(x^{1}\right)^{2}+\left(x^{2}\right)^{2}+\left(x^{3}\right)^{2}}$ and $\hat{x}^{I}:=x^{I} / r$. The phase space is then given by the standard canonical pair $\left(q_{a b}, \pi^{a b}\right)$ satisfying the following fall-off conditions in the cartesian coordinate system: ${ }^{1}$

$$
\begin{aligned}
& q_{I J}=\stackrel{\circ}{q}_{I J}+\frac{h_{I J}(\hat{x})}{r}+O\left(r^{-1-\epsilon}\right), \\
& \pi^{I J}=\frac{p^{I J}(\hat{x})}{r^{2}}+O\left(r^{-2-\epsilon}\right),
\end{aligned}
$$

where $\epsilon>0$ and $h_{I J}$ and $p^{I J}$ are of even and odd parity respectively:

$$
\begin{aligned}
& h_{I J}(-\hat{x})=h_{I J}(\hat{x}), \\
& p^{I J}(-\hat{x})=-p^{I J}(\hat{x}) .
\end{aligned}
$$

${ }^{1} \Sigma, q_{a b}$ and $\pi^{a b}$ are taken to be $C^{\infty}$. A tensor $f$ is $O\left(r^{-\beta}\right)$ (denoted by $O^{\infty}\left(r^{-\beta}\right)$ in [4]) if for $n=0,1, \ldots$, the $n$-th partial derivatives of $f$ in the Cartesian chart, $\partial_{I_{n}} \ldots \partial_{I_{1}} f$, are bounded by $c_{n} r^{-n-\beta}$ for constants $c_{n}$. 
The fall-off conditions ensure the symplectic structure,

$$
\Omega\left(\delta_{1}, \delta_{2}\right)=\frac{1}{16 \pi G} \int_{\Sigma}\left(\delta_{1} q_{a b} \delta_{2} \pi^{a b}-\delta_{2} q_{a b} \delta_{1} \pi^{a b}\right),
$$

is well defined, and allows the existence of non-trivial Poincare generators [3, 4].

We will be dealing with phase space functions $F[q, \pi]$ that are integrals over $\Sigma$ of local functions of $q_{a b}$ and $\pi^{a b}$. The two basic conditions that are required for such expressions are:

(i) $F$ should be finite, i.e., the integral over $\Sigma$ should be convergent

(ii) $F$ should admit a Hamiltonian vector field, i.e. $\delta F=\Omega\left(\delta_{F}, \delta\right) \forall \delta$.

Above $\delta=\left(\delta q_{a b}, \delta \pi^{a b}\right)$ is any variation and $\delta_{F}=\left(\delta_{F} q_{a b}, \delta_{F} \pi^{a b}\right)$ is the Hamiltonian vector field of $F$. Both $\delta$ and $\delta_{F}$ are vector fields in phase space and hence respect the fall-off and parity conditions given above. Condition (ii) encompasses the 'functional differentiability' requirement that $\delta F$ contains no surface terms, and the requirement that the action of $F$ preserves the fall-off and parity conditions. Finally, given two functions $F$ and $G$ satisfying (i) and (ii), their Poisson bracket is defined by $\{F, G\}:=\Omega\left(\delta_{F}, \delta_{G}\right)$.

\section{Constraints and Poincare generators}

In [4] it is shown that the Hamiltonian and diffeomorphism constraints:

$$
\begin{aligned}
H_{0}[N] & :=\frac{1}{16 \pi G} \int_{\Sigma} N\left(q^{-1 / 2}\left(\pi_{a b} \pi^{a b}-\frac{1}{2} \pi^{2}\right)-q^{1 / 2} \mathcal{R}\right), \\
D_{0}[\vec{N}] & :=-\frac{1}{8 \pi G} \int_{\Sigma} N_{a} D_{b} \pi^{a b},
\end{aligned}
$$

satisfy (i) and (ii) when the lapse and shift have the following $r \rightarrow \infty$ asymptotic behavior:

$$
\begin{gathered}
N=S(\hat{x})+O\left(r^{-\epsilon}\right), \\
N^{I}=S^{I}(\hat{x})+O\left(r^{-\epsilon}\right),
\end{gathered}
$$

with

$$
\begin{aligned}
S(-\hat{x}) & =-S(\hat{x}), \\
S^{I}(-\hat{x}) & =-S^{I}(\hat{x}) .
\end{aligned}
$$

$H_{0}[N]$ and $D_{0}[\vec{N}]$ with lapse and shift obeying $\left.(2.8), 2.9\right)$ generate the gauge transformation of the theory.

On the other hand, asymptotic Poincare transformations correspond to lapse and shift satisfying the asymptotic conditions:

$$
\begin{aligned}
N & \rightarrow \alpha+B+\ldots \\
N^{I} & \rightarrow \alpha^{I}+R^{I}+\ldots,
\end{aligned}
$$


where $\alpha, \alpha^{I}$, are constants that represents spacetime translations,

$$
\begin{aligned}
B & =\beta_{I} x^{I} \\
R^{I} & =\beta^{I}{ }_{J} x^{J}
\end{aligned}
$$

with constant $\beta$ 's and $\beta_{J I}=-\beta_{I J}$ represent boost and rotations, and the dots indicates 'pure gauge' terms as in (2.8), (2.9).

In [4] it is shown that the generators of the asymptotic Poincare transformations satisfying (i) and (ii) above are given by:

$$
\begin{aligned}
& H[N]:=H_{0}[N]+\frac{1}{8 \pi G} \oint_{\infty} d S_{d} q^{1 / 2} q^{a[b} q^{c] d}\left(N \stackrel{\circ}{D}_{b} q_{c a}-\stackrel{\circ}{D}_{b} N\left(q_{c a}-\stackrel{\circ}{q}_{c a}\right)\right), \\
& D[\vec{N}]:=D_{0}[\vec{N}]+\frac{1}{8 \pi G} \oint_{\infty} d S_{a} N_{b} \pi^{a b}=\frac{1}{16 \pi G} \int_{\Sigma} \pi^{a b} \mathcal{L}_{\vec{N}} q_{a b},
\end{aligned}
$$

where $\oint_{\infty} \equiv \lim _{r \rightarrow \infty} \oint_{S_{r}}$ with $S_{r}$ the 2 -sphere of radius $r$ with respect to the cartesian system $x^{I}$ and $\stackrel{\circ}{D}$ the derivative compatible with $\stackrel{\circ}{q}_{a b}$.

We emphasize that it is the 'total' $H[N]$ and $D[\vec{N}]$ that satisfy (i) and (ii). The writing of the generators as 'volume plus surface term' is for convenience; each term is not in itself a well defined phase space function. In particular, the surface integrals can be divergent. ${ }^{2}$ On the constraint surface however, finiteness of the generators imply finiteness of the surface integrals, which give then the value of the corresponding Poincare charges (e.g. angular momentum in the case of rotations).

\section{ASHTEKAR-BARBERO VARIABLES}

\section{A. Phase space}

The Cauchy slice $\Sigma$ and the cartesian coordinates $\left\{x^{I}\right\}$ are taken as in the previous section. The canonical pair is now given by an $s u(2)$ connection one-form $A_{a}=A_{a}^{i} \tau_{i}$ and conjugate electric field $E^{a}=E_{i}^{a} \tau_{i}$, with $\tau_{i}, i=1,2,3$ an $s u(2)$ basis satisfying $\left[\tau_{i}, \tau_{j}\right]=\epsilon_{i j k} \tau_{k}$. As will shortly become clear, in order to have a well defined symplectic structure it is necessary that the electric fields asymptote to a fixed densitized triad $E_{i}^{a}$ (whose associated metric is taken to agree with $\stackrel{\circ}{q}_{a b}$ of Eq. (2.1)). We chose this fixed, zeroth order asymptotic electric field to be given by $\stackrel{\circ}{E}_{i}^{I}=\delta_{i}^{I}$. The phase space is then given by pairs $\left(A_{a}^{i}, E_{i}^{a}\right)$ satisfying the analogue of the ADM asymptotic conditions [1]:

$$
\begin{aligned}
& E_{i}^{I}=\stackrel{\circ}{E}_{i}^{I}+\frac{f_{i}^{I}(\hat{x})}{r}+O\left(r^{-1-\epsilon}\right), \\
& A_{I}^{i}=\frac{a_{I}^{i}(\hat{x})}{r^{2}}+O\left(r^{-2-\epsilon}\right),
\end{aligned}
$$

\footnotetext{
${ }^{2}$ This can happen for asymptotic rotations and boosts, and for phase space points outside the constraint surface $H_{0}=D_{0}=0$. This 'off-shell' divergence of surface terms does not occur with the fall-off conditions used in [3], which are schematically of the form $q_{a b}=\stackrel{\circ}{q}_{a b}+($ even $) r^{-1}+O\left(r^{-2}\right)+O\left(r^{-2-\epsilon}\right)$ and $\pi^{a b}=$ (odd) $r^{-2}+O\left(r^{-3}\right)+O\left(r^{-3-\epsilon}\right)$.
} 
with

$$
\begin{aligned}
& f_{i}^{I}(-\hat{x})=f_{i}^{I}(\hat{x}) \\
& a_{I}^{i}(-\hat{x})=-a_{I}^{i}(\hat{x}) .
\end{aligned}
$$

It is not difficult to verify that these fall-offs and parity conditions imply the ADM ones described in the previous section. The fall-off conditions ensure the symplectic structure,

$$
\Omega\left(\delta_{1}, \delta_{2}\right):=\frac{1}{8 \pi G \gamma} \int_{\Sigma}\left(\delta_{1} A_{a}^{i} \delta_{2} E_{i}^{a}-\delta_{2} A_{a}^{i} \delta_{1} E_{i}^{a}\right),
$$

is well defined. We now see the need to keep fixed the zeroth order electric field in (3.1): Had we allowed for all possible $S U(2)$-rotated $E_{i}^{a}$ 's (so that the asymptotic metric still satisfies (2.1)), we could not have ensured convergence of the integral (3.5).

Below we will be dealing with phase space functions $F[A, E]$ that are integrals over $\Sigma$ of local functions of $A_{a}^{i}$ and $E_{i}^{a}$. Such functions will be required to satisfy the conditions (i) and (ii) described at the end of section II 1 (now with respect to the sympectic form (3.5)).

\section{B. Constraints}

\section{Gauss Constraint}

In connection variables, there appears the additional Gauss law constraint

$$
G[\Lambda]:=\frac{1}{8 \pi G \gamma} \int_{\Sigma} \Lambda^{i} \mathcal{D}_{a} E_{i}^{a},
$$

where $\mathcal{D}_{a}$ is the covariant derivative associated to the connection $A_{a}^{i}$, acting as $\mathcal{D}_{a} f_{i}=$ $\partial_{a} f_{i}+\epsilon_{i j k} A_{a}^{j} f^{k}$. Since both terms in $\mathcal{D}_{a} E_{i}^{a}$ fall off as (odd) $r^{-2}+O\left(r^{-2-\epsilon}\right)$, the minimal condition on the multiplier $\Lambda^{i}$ ensuring convergence of the integral is:

$$
\Lambda^{i}=\frac{\lambda^{i}(\hat{x})}{r}+O\left(r^{-1-\epsilon}\right)
$$

with

$$
\lambda^{i}(-\hat{x})=\lambda^{i}(\hat{x}) .
$$

We now verify that with this fall-off and parity condition, $G[\Lambda]$ also satisfies (ii):

$$
\begin{aligned}
\delta G & =\frac{1}{8 \pi G \gamma} \int_{\Sigma} \Lambda^{i}\left(\partial_{a} \delta E_{i}^{a}+\epsilon_{i j k} A_{a}^{j} \delta E_{k}^{a}+\epsilon_{i j k} \delta A_{a}^{j} E_{k}^{a}\right) \\
& =\frac{1}{8 \pi G \gamma} \int_{\Sigma}\left(\delta_{\Lambda} A_{a}^{i} \delta E_{i}^{a}-\delta A_{a}^{i} \delta_{\Lambda} E_{i}^{a}\right) \equiv \Omega\left(\delta_{\Lambda}, \delta\right)
\end{aligned}
$$

where

$$
\begin{aligned}
& \delta_{\Lambda} A_{a}^{i}=-\partial_{a} \Lambda^{i}+\epsilon_{i j k} \Lambda^{j} A_{a}^{k}=-\mathcal{D}_{a} \Lambda^{i} \\
& \delta_{\Lambda} E_{i}^{a}=\epsilon_{i j k} \Lambda^{j} E_{k}^{a} .
\end{aligned}
$$


In going from (3.9) to 3.10 , we performed the integration by parts:

$$
\int_{\Sigma} \Lambda^{i} \partial_{a} \delta E_{i}^{a}=\oint_{\infty} d S_{a} \Lambda^{i} \delta E_{i}^{a}-\int_{\Sigma} \partial_{a} \Lambda^{i} \delta E_{i}^{a}=-\int_{\Sigma} \partial_{a} \Lambda^{i} \delta E_{i}^{a}
$$

where the surface term being (odd) (even) $r^{-1}$ (even) $r^{-1}+O\left(r^{-2-\epsilon}\right)$ vanishes. It is easy to verify that (3.11) and (3.12) preserve the fall-off and parity conditions, and hence is a well defined phase space variation. Finally, the relation $\left\{G[\Lambda], G\left[\Lambda^{\prime}\right]\right\}=G\left[\left[\Lambda, \Lambda^{\prime}\right]\right]$ can be verified thanks to the vanishing of the surface term:

$$
\oint_{\infty} d S_{a} \epsilon_{i j k} E_{i}^{a} \Lambda_{j} \Lambda_{k}^{\prime}=0
$$

as implied by the fall-off and parity condition (3.7).

We now show that the leading term in (3.7) is crucial for the recovery of ADM phase space in that it accounts for 'pure $S U(2)$ gauge' components of the $r^{-1}$ term of the triad (3.1). The doubly densitized inverse metric is given by $\tilde{\tilde{q}}^{I J}=E_{i}^{I} E_{i}^{J}=\stackrel{\circ}{E}_{i}^{I} \stackrel{\circ}{E}_{i}^{J}+2 \stackrel{\circ}{E}_{i}^{(I} f_{i}^{J)} / r+O\left(r^{-1-\epsilon}\right)$ from which it follows that

$$
q_{I J}=\stackrel{\circ}{E}_{i}^{I} \stackrel{\circ}{E}_{i}^{J}-2 \stackrel{\circ}{E}_{i}^{(I} f_{i}^{J)} / r+O\left(r^{-1-\epsilon}\right) .
$$

Define $f_{I J}:=-2 \stackrel{\circ}{E}_{i}^{I} f_{i}^{J} \equiv-2 f_{I}^{J}$. Equating 3.15 with 2.1 we conclude that the $r^{-1}$ term of the metric is given by: $h_{I J}=f_{(I J)}$. From this perspective $f_{[I J]}$ appear as 'pure gauge' components.

On the other hand, the variation of $f_{I J}$ under $S U(2)$ gauge transformation can be found by substituting (3.1) and (3.7) in (3.12):

$$
\delta_{\Lambda} f_{i}^{I}=\epsilon_{i j k} \lambda^{j} \stackrel{\circ}{E}_{k}^{I} \Longrightarrow \delta_{\Lambda} f_{I J}=2 \epsilon_{I J k} \lambda^{k},
$$

which is in agreement with the previous 'pure gauge' interpretation of $f_{[I J]}$.

In the following sections we will often encounter Gauss constraints (3.6) smeared with phase space dependent multipliers. We now verify properties (i) and (ii) are still satisfied in such cases. For $\Lambda^{i}=\Lambda^{i}(A, E)$ satisfying (3.7) and (3.8), finiteness follows by the some fall-off/parity argument given before. From (3.10) it follows that the variation of $G[\Lambda]$ is now given by

$$
\delta G[\Lambda]=\Omega\left(\delta_{\Lambda(A, E)}, \delta\right)+\frac{1}{8 \pi G \gamma} \int_{\Sigma} \delta \Lambda^{i}(A, E) \mathcal{D}_{a} E_{i}^{a} .
$$

If $\Lambda^{i}=\Lambda^{i}(A, E)$ does not depend on derivatives of $A_{a}^{i}$ or $E_{i}^{a}$, then (3.17) is already differentiable. Otherwise one needs to integrate by parts the second term in (3.17) to obtain an integrand that does not depend on derivatives of the variations $\delta A_{a}^{i}, \delta E_{i}^{a}$. We now argue that the corresponding surface terms will always be (odd) $r^{-2}+O\left(r^{-2-\epsilon}\right)$ and hence vanish. Consider a term in $\delta \Lambda^{i}$ of the form $F_{a i j}^{b}[A, E] \partial_{b} \delta E_{j}^{a}$. Since $\delta \Lambda^{i}=($ even $) r^{-1}$ and $\partial_{b} \delta E_{j}^{a}=($ odd $) r^{-2}$ it follows that $F_{a i j}^{b}=($ odd $) r$. The surface term will then be $d S_{b} F_{a i j}^{b} \delta E_{j}^{a} \mathcal{D}_{c} E_{i}^{c}=($ odd $) r^{-2}+O\left(r^{-2-\epsilon}\right)$. A similar argument shows that the surface term coming from a variation involving a derivative of $\delta A_{a}^{i}$ also vanishes. The argument may also be extended to allow for derivatives of higher order, but the above considerations are enough for our purposes since all phase space dependent multipliers we encounter depend at most 
on first derivatives of the canonical variables. Thus, we conclude that the Gauss constraint with phase space dependent multiplier satisfying (3.7) and (3.8) is differentiable. Finally, it is easy to verify from the above expressions that the contribution to the Hamiltonian vector field coming from the second term in (3.17) also preserves the fall-off and parity conditions.

As a final note, we point out that the alternative expression of the Gauss constraint obtained by integration by parts in (3.6) leads to

$$
G[\Lambda]=-\frac{1}{8 \pi G \gamma} \int_{\Sigma} E_{i}^{a} \mathcal{D}_{a} \Lambda^{i}+\frac{1}{8 \pi G \gamma} \oint_{\infty} d S_{a} E_{i}^{a} \Lambda_{i} .
$$

Whereas the full $G[\Lambda]$ is well defined, the two terms in the RHS (3.18) are not necessarily well defined by themselves. Indeed, it can be easily seen that the fall-offs (3.7) do not ensure convergence of the surface term in (3.18).

\section{Hamiltonian and diffeomorphism constraints}

We start with the following form of the Hamiltonian and diffeomorphism constraints [12]:

$$
\begin{aligned}
H_{0}[N]: & :=\frac{1}{16 \pi G} \int_{\Sigma} N\left(\epsilon_{i j k} F_{a b}^{i} E_{j}^{a} E_{k}^{b}-2\left(1+\gamma^{2}\right) K_{[a}^{i} K_{b]}^{j} E_{i}^{a} E_{j}^{b}\right), \\
D_{0}[\vec{N}]: & :=\frac{1}{8 \pi G \gamma} \int_{\Sigma} E_{i}^{a} \mathcal{L}_{\vec{N}} A_{a}^{i},
\end{aligned}
$$

where $F_{a b}^{i}:=\partial_{a} A_{b}^{i}-\partial_{b} A_{a}^{i}+\epsilon_{i j k} A_{a}^{j} A_{b}^{k}, K_{a}^{i}:=\gamma^{-1}\left(A_{a}^{i}-\Gamma_{a}^{i}\right)$ and $N$ of density weight -1 . The relation between the constraints $H_{0}$ and $D_{0}$ of this section and those of section [II will be described in section IV.

The minimal conditions for (3.19) and (3.20) to be finite are as in the ADM case:

$$
\begin{aligned}
N & =S(\hat{x})+O\left(r^{-\epsilon}\right), \\
N^{I} & =S^{I}(\hat{x})+O\left(r^{-\epsilon}\right),
\end{aligned}
$$

with $S$ and $S^{I}$ odd. It is easy to verify that $D_{0}$ and the first term in $H_{0}$ satisfy (ii). We now argue the second, 'KKEE' term in $H_{0}$ also satisfies (ii). Under variations of this term, the potentially problematic surface contribution come from derivatives of the triad in $\Gamma_{a}^{i}$. Schematically:

$$
\left(\int N E E K \delta \Gamma\right)_{\mathrm{Bdy}}=\oint N E E K \delta E=0,
$$

where the vanishing occurs since the integrand of the surface term falls off as $r^{-3}$. Finally, it is easy to verify that the contribution from the KKEE piece to the Hamiltonian vector field preserves the fall off and parity conditions.

$G[\Lambda], H_{0}[N]$ and $D_{0}[\vec{N}]$ with multipliers satisfying the conditions above are the constraints/gauge generators of the theory. 


\section{Poincare generators}

We now want to extend $H_{0}$ and $D_{0}$ in order to obtain well defined generators for lapse and shift corresponding to asymptotic Poincare transformations:

$$
\begin{aligned}
N & \rightarrow \alpha+B+\ldots \\
N^{I} & \rightarrow \alpha^{I}+R^{I}+\ldots,
\end{aligned}
$$

with $B=\beta_{I} x^{I}, R^{I}=\beta_{J}^{I} x^{J}$ as in section II 2 and the dots indicate gauge terms (3.21), (3.22). Following the strategy of [3] we will start by adding surface terms that cancel the unwanted boundary contribution of the variations of $H_{0}$ and $D_{0}$.

First, we notice that the 'KKEE' term of the Hamiltonian constraint (3.19) is still well defined for the more general lapse (3.24): The leading term in the lapse is now (odd) $r$ so that NKKEE $\sim($ odd $) r^{-3}+O\left(r^{-3-\epsilon}\right)$ and the integral converges; the potentially problematic surface term (3.23) is now (odd) $r^{-2}+O\left(r^{-2-\epsilon}\right)$ and again vanishes. It is also easy to verify that the corresponding Hamiltonian vector field preserves the fall off and parity conditions.

Thus, the surface terms that cancel the unwanted boundary contributions are the same as in the self-dual formulation [6]:

$$
\begin{aligned}
& H_{1}[N]:=H_{0}[N]-\frac{1}{8 \pi G} \oint_{\infty} d S_{a} N \epsilon_{i j k} A_{b}^{i} E_{j}^{a} E_{k}^{b}, \\
& D_{1}[\vec{N}]:=D_{0}[\vec{N}]-\frac{1}{8 \pi G \gamma} \oint_{\infty} d S_{a} N^{a} A_{b}^{i} E_{i}^{b}=-\frac{1}{8 \pi G \gamma} \int_{\Sigma} A_{a}^{i} \mathcal{L}_{\vec{N}} E_{i}^{a} .
\end{aligned}
$$

For the case of asymptotic spacetime translations (so that $B=0$ and $R^{I}=0$ in $(3.24)$ and (3.25),$H_{1}$ and $D_{1}$ yield well defined phase space generators which agree with the ADM ones [1, 6]. This result will be recovered as a particular case of the general Poincare generators discussed below.

At a formal level, even for boosts and rotations the variations of $H_{1}$ and $D_{1}$ have no surface terms. However $H_{1}$ and $D_{1}$ are no longer guaranteed to be finite.

As pointed out in [1], the reason these functions are not well defined for nonzero rotations or boosts becomes clear when one realizes their action would change the zeroth order part of the triad and thus map us out of phase space. This suggests one should modify the expression by adding a suitable Gauss piece in such a way that the zeroth order part of the triad is kept fixed. In the following we implement this idea.

\section{Rotations}

It will be convenient to work with the last expression in (3.27). When the shift has a nonzero rotation at infinity, the integrand has the following asymptotic behavior:

$$
-A_{I}^{i} \mathcal{L}_{\vec{N}} E_{i}^{I}=A_{I}^{i} E_{i}^{J} \partial_{J} N^{I}+\ldots \underset{r \rightarrow \infty}{\longrightarrow} A_{I}^{i} \stackrel{\circ}{E}_{i}^{J} \beta_{J}^{I}+(\text { odd }) r^{-3}+O\left(r^{-3-\epsilon}\right) .
$$

The first term falls off as (odd) $r^{-2}+O\left(r^{-2-\epsilon}\right)$ and thus we cannot ensure converge of the integral. This is the same term responsible for rotating the zeroth order part of the triad. 
In order to compensate, we subtract an appropriate Gauss term $G\left[\Lambda_{R}\right]$ with

$$
\Lambda_{R}^{i}=\AA_{R}^{i}+\Lambda^{i},
$$

where

$$
\stackrel{\circ}{\Lambda}_{R}^{i}:=-\frac{1}{2} \epsilon_{i j k} \stackrel{\circ}{E}_{I}^{j} \stackrel{\circ}{E}_{k}^{J} \beta^{I}{ }_{J}=\frac{1}{2} \epsilon_{i j k} \stackrel{\circ}{E}_{a}^{j} \mathcal{L}_{\vec{R}} \stackrel{\circ}{E}_{k}^{a}
$$

is a constant $\left(\partial_{a} \Lambda_{R}^{i}=0\right)$, zeroth order term, and $\Lambda^{i}$ a 'pure gauge' multiplier as in (3.7), (3.8).

By subtracting $G\left[\Lambda_{R}\right]$ from $D_{1}[\vec{N}]$ we cancel the term responsible for the divergence in (3.28). This also introduces a new divergent and non-differentiable piece which is removed by including an appropriate boundary term. The final expression is:

$$
D[\vec{N}]:=D_{1}[\vec{N}]-G\left[\Lambda_{R}\right]+\frac{1}{8 \pi G \gamma} \oint_{\infty} d S_{a} E_{i}^{a} \AA_{R}^{i}
$$

We now verify (3.31) satisfies (i) and (ii). In section IV] we show (3.31) agrees, modulo pure gauge Gauss constraint terms, with ADM and Thiemann's expressions.

To show finiteness, we write (3.31) as a volume integral:

$$
D[\vec{N}]=\frac{1}{8 \pi G \gamma} \int_{\Sigma}\left(-A_{a}^{i} \mathcal{L}_{\vec{N}} E_{i}^{a}-\epsilon_{i j k} \Lambda_{R}^{i} A_{a}^{j} E_{k}^{a}-\left(\Lambda_{R}^{i}-\AA_{R}^{i}\right) \partial_{a} E^{i}\right),
$$

where we used that $\partial_{a}\left(\AA_{R}^{i} E_{i}^{a}\right)=\AA_{R}^{i} \partial_{a} E_{i}^{a}$. By construction the first two terms in 3.32 combine to give a convergent fall-off:

$$
-A_{I}^{i} \mathcal{L}_{\vec{N}} E_{i}^{I}-\epsilon_{i j k} \Lambda_{R}^{i} A_{I}^{j} E_{k}^{I}=(\text { odd }) r^{-3}+O\left(r^{-3-\epsilon}\right),
$$

where the cancelation of the would-be divergent terms can be explicitly verified by substituting (3.30) in (3.33). The last term in (3.32) is clearly convergent. We thus conclude (3.31) is finite. Let us now verify (ii). As mentioned earlier the first term in $(3.32)$ is functional differentiable. By the same arguments given for the differentiability of the Gauss constraint one finds that the last term in (3.32) is also functional differentiable. The total variation can finally be written as

$$
\delta D[\vec{N}]=\Omega\left(\delta_{D[\vec{N}]}, \delta\right),
$$

with

$$
\begin{aligned}
& \delta_{D[\vec{N}]} A_{a}^{i}=\mathcal{L}_{\vec{N}} A_{a}^{i}-\delta_{\Lambda_{R}} A_{a}^{i} \\
& \delta_{D[\vec{N}]} E_{i}^{a}=\mathcal{L}_{\vec{N}} E_{i}^{a}-\delta_{\Lambda_{R}} E_{i}^{a},
\end{aligned}
$$

where $\delta_{\Lambda_{R}}$ is given by $(3.11),(3.12)$ with $\Lambda=\Lambda_{R}$. It is easy to verify that $\delta_{D[\vec{N}]}$ preserves the falloff and parity conditions. ${ }^{3}$

Note that in the above discussion the shift was of the general type 3.25 . If $R^{I}=0$ then $\Lambda_{R}^{i}=0$ and we recover the generator of translations 3.27) (up to a possible 'pure gauge'

\footnotetext{
${ }^{3}$ Each term in 3.35 is a well defined variation. For 3.36 only the total expression is a valid variation,
} but not each term independently (except when $R^{I}=0$ ). 
Gauss term).

We conclude the section by verifying $(3.31)$ is $S U(2)$ gauge invariant in the sense that it weakly commutes with the Gauss constraint. Using Eqns. (3.11), (3.12), (3.35), (3.36), one finds:

$$
\{D[\vec{N}], G[\Lambda]\}=G\left[\mathcal{L}_{\vec{N}} \Lambda-\left[\Lambda_{R}, \Lambda\right]\right]-\frac{1}{8 \pi G \gamma} \oint_{\infty} d S_{a} E_{i}^{a}\left(\mathcal{L}_{\vec{N}} \Lambda^{i}-\left[\Lambda_{R}, \Lambda\right]^{i}\right)
$$

The multiplier of the Gauss term in the RHS of (3.37) is (even) $r^{-1}+O\left(r^{-1-\epsilon}\right)$ and hence satisfies the conditions of section IIIB 1. We now show that the surface term in (3.37) vanishes. The first term can be written as:

$$
\oint_{\infty} d S_{a} E_{i}^{a} \mathcal{L}_{\vec{N}} \Lambda^{i}=-\oint_{\infty} d S_{a} \mathcal{L}_{\vec{R}} \stackrel{\circ}{i}_{i}^{a} \Lambda^{i}
$$

where we used the fact that $\mathcal{L}_{\vec{N}} \Lambda=($ even $) r^{-1}+O\left(r^{-1-\epsilon}\right)$ and Eq. A8. For the second term we have,

$$
\begin{aligned}
-\oint_{\infty} d S_{a} E_{i}^{a}\left[\Lambda_{R}, \Lambda\right]^{i} & =-\oint_{\infty} d S_{a} \epsilon_{i j k} \stackrel{\circ}{E}_{i}^{a} \Lambda_{R}^{j} \Lambda^{k} \\
& =-\frac{1}{2} \oint_{\infty} d S_{a} \epsilon_{i j k} \epsilon_{j m n}{\stackrel{\circ}{E_{i}^{a}}}^{a} \stackrel{\circ}{E}_{b}^{m} \mathcal{L}_{\vec{R}} \stackrel{\circ}{E}_{n}^{b} \Lambda^{k} \\
& =\frac{1}{2} \oint_{\infty} d S_{a}\left(\mathcal{L}_{\vec{R}} \stackrel{\circ}{E}_{i}^{a} \Lambda^{i}-\stackrel{\circ}{E}_{b}^{i} \mathcal{L}_{\vec{R}} \stackrel{\circ}{E}_{j}^{b} \stackrel{\circ}{E}_{j}^{a} \Lambda^{i}\right) \\
& =\oint_{\infty} d S_{a} \mathcal{L}_{\vec{R}} \stackrel{\circ}{E}_{i}^{a} \Lambda^{i},
\end{aligned}
$$

where we used the fact that $\left[\Lambda_{R}, \Lambda\right]=($ even $) r^{-1}+O\left(r^{-1-\epsilon}\right)$ and $\mathcal{L}_{\vec{R}} \stackrel{\circ}{E}_{j}^{b} \stackrel{\circ}{E}_{j}^{a}=-\stackrel{\circ}{E}_{j}^{b} \mathcal{L}_{\vec{R}} \stackrel{\circ}{E}_{j}^{a}$. The two terms cancel each other and we conclude that $D[\vec{N}]$ weakly commutes with the Gauss constraint.

\section{Boosts}

Expressing the boundary term in (3.26) as a volume integral, one can isolate the divergent term:

$$
H_{1}[N]=-\frac{1}{8 \pi G} \int_{\Sigma} \stackrel{\circ}{D}_{a} N \epsilon_{i j k} A_{b}^{i} E_{j}^{a} E_{k}^{b}+\ldots
$$

where the dots represents terms whose integral is convergent for $N \rightarrow \beta_{I} x^{I}$. The divergent piece can be removed by subtracting a Gauss term $G\left[\Lambda_{B}\right]$ with

$$
\Lambda_{B}^{i}=\stackrel{\circ}{\Lambda}_{B}^{i}+\Lambda^{i}
$$

where

$$
\stackrel{\circ}{\Lambda}_{B}^{i}:=\beta_{I} \stackrel{\circ}{E}_{i}^{I}=\stackrel{\circ}{D}_{a} B \stackrel{\circ}{E}_{i}^{a}
$$

is a constant, zeroth order term and $\Lambda^{i}$ a 'pure gauge' multiplier as in (3.7), (3.8). As in the case of rotations, the Gauss term introduces a divergent and non-differentiable piece that 
can be removed by an appropriate surface term. The final expression is:

$$
H[N]:=H_{1}[N]-\gamma G\left[\Lambda_{B}\right]+\frac{1}{8 \pi G} \oint_{\infty} d S_{a} E_{i}^{a} \stackrel{\Lambda}{B}_{B}^{i}
$$

We now verify the expression satisfies (i) and (ii). In section IV we show (3.46) agrees, modulo pure gauge Gauss constraint terms, with ADM and Thiemann's expressions.

As we did for the rotations, let us express 3.46 as a volume integral $H[N]=(8 \pi G)^{-1} \int_{\Sigma} \rho$ with:

$$
\rho=-N \epsilon_{i j k} A_{b} \stackrel{\circ}{D}_{a}\left(E_{j}^{a} E_{k}^{b}\right)-\stackrel{\circ}{D}_{a} N \epsilon_{i j k} A_{b}^{i} E_{j}^{a} E_{k}^{b}-\epsilon_{i j k} \Lambda_{B}^{i} A_{b}^{j} E_{k}^{b}-\left(\Lambda_{B}^{i}-\stackrel{\circ}{\Lambda}_{B}^{i}\right) \partial_{a} E^{i}+\ldots
$$

The dots indicate the 'NAAEE' term coming from the non-abelian part of $F_{a b}$ and the 'Lorentzian' 'NKKEE' term. The former is manifestly finite and differentiable. The latter is also finite and differentiable by the discussion given in the beginning of IIIC. We then focus on the terms displayed in (3.47). The first and last terms are easily verified to give a finite and differentiable contribution to $H$. By construction, the potentially divergent contributions from the second and third term cancel out, as can be verified by using (3.24) and $(3.45)$. We conclude that $H[N]$ is finite and differentiable. One can further verify that all possible contributions to the Hamiltonian vector field are such that they preserve the fall-off and parity conditions so that $H[N]$ satisfies (ii).

We finally note that if $B=0$, then $\Lambda_{B}^{i}=0$ and we recover the generator of time translations (3.26), up to a possible 'pure gauge' Gauss term.

\section{COMPARISON WITH ADM AND THIEMANN'S EXPRESSIONS}

\section{A. Diffeomorphism constraint, asymptotic translations and rotations}

We give a quick re-derivation of Thiemann's expressions based on the ADM ones, and show they coincide (up to pure gauge Gauss terms) with the expressions from last section. We start with the ADM generator (2.17):

$$
D_{\mathrm{ADM}}[\vec{N}]=\frac{1}{16 \pi G} \int_{\Sigma} \pi^{a b} \mathcal{L}_{\vec{N}} q_{a b}
$$

with a general shift of the form 2.13), and seek to rewrite it in terms of $\left(A_{a}^{i}, E_{i}^{a}\right)$ variables. A small computation shows the integrand in (4.1) can be rewritten as,

$$
\pi^{a b} \mathcal{L}_{\vec{N}} q_{a b}=-2 q^{-1 / 2} K_{a b} \mathcal{L}_{\vec{N}} q q^{a b}
$$

where we used $\pi^{a b}=q^{1 / 2}\left(K^{a b}-K q^{a b}\right)$. Performing the substitution,

$$
\begin{aligned}
q q^{a b} & =E_{i}^{a} E_{i}^{b}, \\
q^{-1 / 2} K_{a b} & =E_{(a}^{j} K_{b)}^{j},
\end{aligned}
$$


in (4.2), the ADM generator (4.1) becomes:

$$
D_{\mathrm{ADM}}[\vec{N}]=-\frac{1}{8 \pi G} \int_{\Sigma}\left(K_{a}^{i} \mathcal{L}_{\vec{N}} E_{i}^{a}+\epsilon_{i j k} \Lambda_{\vec{N}}^{i} K_{a}^{j} E_{k}^{a}\right)
$$

where we have defined

$$
\Lambda_{\vec{N}}^{i}:=\frac{1}{2} \epsilon_{i j k} E_{a}^{j} \mathcal{L}_{\vec{N}} E_{k}^{a}
$$

Finally, substituting $K_{a}^{i}=\gamma^{-1}\left(A_{a}^{i}-\Gamma_{a}^{i}\right)$ we recover the expression given in [1]:

$$
D_{\mathrm{ADM}}[\vec{N}]=D_{1}[\vec{N}]-G\left(\Lambda_{\vec{N}}\right)+\frac{1}{8 \pi G \gamma} \int_{\Sigma} \Gamma_{a}^{i} \mathcal{L}_{\vec{N}} E_{i}^{a},
$$

with $D_{1}$ and $G$ given by (3.27) and (3.6) respectively. As we shall see, the last term in (4.7) can be written as a surface term. In appendix A3 we display this surface term in the form given in [1].

In the following we discuss asymptotic rotations and translations separately. We will write the last term in (4.7) in a way that will facilitate the comparison with the expressions of section III.

\section{Rotations}

Consider the case where $\alpha^{I}=0$ so that ,

$$
N^{I}=R^{I}+S^{I}+O\left(r^{-\epsilon}\right),
$$

with $R^{I}$ as in 2.15) and $S^{I}$ as in 2.11). The last term in the RHS of 4.7) can be shown to be a pure boundary term as follows. First integrate by parts,

$$
\int \Gamma_{a}^{i} \mathcal{L}_{\vec{N}} E_{i}^{a}=\oint d S_{a} N^{a} \Gamma_{b}^{i} E_{i}^{b}-\int E_{i}^{a} \mathcal{L}_{\vec{N}} \Gamma_{a}^{i}
$$

The surface integral vanishes since $d S_{a} N^{a}=($ even $)+O\left(r^{-\epsilon}\right), \Gamma_{b}^{i}=($ odd $) r^{-2}$ and $E_{i}^{b}$ as in (3.1). For the second integral we use the identity (see appendix A 1),

$$
E_{i}^{a} \delta \Gamma_{a}^{i}=-\frac{1}{2} \epsilon_{i j k} \partial_{a}\left(E_{i}^{a} E_{b}^{j} \delta E_{k}^{b}\right)
$$

to write it as a boundary term:

$$
\begin{aligned}
-\int E_{i}^{a} \mathcal{L}_{\vec{N}} \Gamma_{a}^{i} & =\frac{1}{2} \oint_{\infty} d S_{a} \epsilon_{i j k} E_{i}^{a} E_{b}^{j} \mathcal{L}_{\vec{N}} E_{k}^{b} \\
& =\oint_{\infty} d S_{a} E_{i}^{a} \Lambda_{\vec{N}}^{i},
\end{aligned}
$$

where in the second equality we used the definition of $\Lambda_{\vec{N}}(4.6)$. We thus obtain:

$$
D_{\mathrm{ADM}}[\vec{N}]=D_{1}[\vec{N}]-G\left(\Lambda_{\vec{N}}\right)+\frac{1}{8 \pi G \gamma} \oint_{\infty} d S_{a} E_{i}^{a} \Lambda_{\vec{N}}^{i}
$$


Expression (4.13) resembles that of the generator (3.31) given in the previous section. Choosing for simplicity $\Lambda_{R}=\AA_{R}$ in 3.31 , the difference between the two is:

$$
D[\vec{N}]-D_{\mathrm{ADM}}[\vec{N}]=G\left[\lambda_{\vec{N}}\right]-\frac{1}{8 \pi G \gamma} \oint_{\infty} d S_{a} E_{i}^{a} \lambda_{\vec{N}}^{i}
$$

with

$$
\lambda_{\vec{N}}^{i}:=\Lambda_{\vec{N}}^{i}-\AA_{R}^{i}=(\text { even }) r^{-1}+O\left(r^{-1-\epsilon}\right) .
$$

In appendix $\mathrm{B}$ we show that

$$
\oint_{\infty} d S_{a} E_{i}^{a} \lambda_{\vec{N}}^{i}=0
$$

and hence the difference 4.14) is a 'pure gauge' Gauss term $G\left(\lambda_{\vec{N}}\right)$ (with phase space dependent multiplier).

\section{Translations}

For completeness we re-derive the result that for asymptotic translations the generator (3.27) coincides, modulo a Gauss term, with the ADM generator (4.7). Since the considerations from the previous section already account for the $S^{I}$ and $O\left(r^{-\epsilon}\right)$ terms in the shift (see appendix $\mathrm{B}$ ), we now restrict attention to shifts of the form

$$
N^{I}=\alpha^{I}+O\left(r^{-1-\epsilon}\right) .
$$

The surface term that was dropped in $(4.9)$ no longer vanishes and so the last term in the RHS of 4.7) now becomes

$$
\int_{\Sigma} \Gamma_{a}^{i} \mathcal{L}_{\vec{N}} E_{i}^{a}=\oint d S_{a}\left(N^{a} \Gamma_{b}^{i} E_{i}^{b}+E_{i}^{a} \Lambda_{\vec{N}}^{i}\right)
$$

For the first term in 4.18 we write:

$$
\Gamma_{b}^{i} E_{i}^{b}=-\frac{1}{2} \epsilon_{i j k} E_{i}^{b} E_{c}^{j} \stackrel{\circ}{D}_{b} E_{k}^{c}
$$

(this follows from Eq. A11 by noting that the additional $\left(D_{b}-\stackrel{\circ}{D}_{b}\right)$ contribution vanishes). For the second term we write

$$
\Lambda_{\vec{N}}^{i}=\frac{1}{2} \epsilon_{i j k} E_{c}^{j} N^{b} \stackrel{\circ}{D}_{b} E_{k}^{c}+O\left(r^{-2-\epsilon}\right)
$$

where we used that $\stackrel{\circ}{D}_{a} N^{b}=O\left(r^{-2-\epsilon}\right)$ for the shift 4.17$)$. Since $\stackrel{\circ}{D}_{a} E_{i}^{b}=($ odd $) r^{-2}$, the triads in 4.19) and 4.20 that are not being derivated can be set to their zeroth order value. Defining

$$
X^{a}:=\frac{1}{2} \epsilon_{i j k} \stackrel{\circ}{E}_{i}^{a} \stackrel{\circ}{E}_{c}^{j} E_{k}^{c}
$$


the surface integral 4.18 becomes

$$
\oint d S_{a}\left(-N^{a} \stackrel{\circ}{D}_{b} X^{b}+N^{b} \stackrel{\circ}{D}_{b} X^{a}\right)=\oint d S_{a}\left(-N^{a} \stackrel{\circ}{D}_{b} X^{b}+\mathcal{L}_{\vec{N}} X^{a}\right)=0
$$

where we used Eq. (A8). Thus, the surface term vanishes and the generators (4.7) and (3.27) differ by a pure gauge Gauss term.

\section{B. Hamiltonian constraint, asymptotic time translations and boosts}

We start with a quick re-derivation of the Hamiltonian in Ashtekar-Barbero variables in order to ensure no further subtleties arise from the 'KKEE' term. Let

$$
H_{\mathrm{ADM}}\left[N_{\mathrm{ADM}}\right]=H_{\mathrm{ADM}}^{0}\left[N_{\mathrm{ADM}}\right]+S\left[N_{\mathrm{ADM}}\right]
$$

be the full ADM Hamiltonian (2.16) with $H_{\mathrm{ADM}}^{0}\left[N_{\mathrm{ADM}}\right]$ given by Eq. (2.6) and $S\left[N_{\mathrm{ADM}}\right]$ the surface term in (2.16). The lapse is taken to be $N_{\text {ADM }}=q^{1 / 2} N$ with $N$ the density weight -1 lapse satisfying (3.24).

For the integrand of $H_{\mathrm{ADM}}^{0}$ we use the identities (see for instance [11]):

$$
\begin{aligned}
-q R & =\epsilon_{i j k} F_{a b}^{i} E_{j}^{a} E_{k}^{b}+2 D_{a}\left(E_{i}^{a} \mathcal{D}_{b} E_{i}^{b}\right)-2 \gamma^{2} K_{[a}^{i} K_{b]}^{j} E_{i}^{a} E_{j}^{b} \\
\pi_{a b} \pi^{a b}-\frac{1}{2} \pi^{2} & =-2 K_{[a}^{i} K_{b]}^{j} E_{i}^{a} E_{j}^{b}+\frac{1}{2 \gamma^{2}} \mathcal{D}_{a} E_{i}^{a} \mathcal{D}_{b} E_{i}^{b}
\end{aligned}
$$

where $D_{a}$ is the derivative compatible with $q_{a b}(4.3)$. For the surface term $S\left[N_{\mathrm{ADM}}\right]$ we use the result derived in [1]:

$$
S\left[q^{1 / 2} N\right]=\frac{1}{8 \pi G} \oint_{\infty} d S_{a}\left(-N E_{i}^{a} \partial_{b} E_{i}^{b}+D_{b} N E_{i}^{b}\left(E_{i}^{a}-\stackrel{\circ}{E}_{i}^{a}\right)\right)
$$

We now use 4.24, 4.25, 4.26) to express 4.23) in $\left(A_{a}^{i}, E_{i}^{a}\right)$ variables. Subtracting for convenience the "pure gauge' Gauss piece arising from the second term in 4.25) and integrating by parts the middle term in 4.24, , one obtains:

$$
\begin{aligned}
H_{\mathrm{ADM}}^{\prime}\left[q^{1 / 2} N\right] & :=H_{\mathrm{ADM}}\left[q^{1 / 2} N\right]-(4 \gamma)^{-1} G\left[N \mathcal{D}_{a} E^{a}\right] \\
& =H_{1}[N]-\gamma G\left[\Lambda_{N}\right]+\frac{1}{8 \pi G} \oint_{\infty} d S_{a} \Lambda_{N}^{i}\left(E_{i}^{a}-\stackrel{\circ}{E}_{i}^{a}\right),
\end{aligned}
$$

with $H_{1}$ given by 3.26$), G$ as in $(3.6)$ and

$$
\Lambda_{N}^{i}:=D_{a} N E_{i}^{a}
$$

Expression (4.28) corresponds to the one given in [1, written in a way that will facilitate comparison with $H[N]$ 3.46. Subtracting $0=\oint_{\infty} d S_{a} \stackrel{\circ}{\Lambda}_{B}^{i} \stackrel{\circ}{E}_{i}^{a}$ in $H[N]$ so that the surface term in 3.46 involves the difference $\left(E_{i}^{a}-{\stackrel{\circ}{E_{i}^{a}}}^{a}\right)$ as in 4.28, we find:

$$
H_{\mathrm{ADM}}^{\prime}\left[q^{1 / 2} N\right]-H[N]=-\gamma G\left[\Lambda_{N}-\Lambda_{B}\right]+\frac{1}{8 \pi G} \oint_{\infty} d S_{a}\left(\Lambda_{N}^{i}-\stackrel{\circ}{\Lambda}_{B}^{i}\right)\left(E_{i}^{a}-\stackrel{\circ}{E}_{i}^{a}\right) .
$$


The difference $\Lambda_{N}^{i}-\Lambda_{B}^{i}$ can be seen to be (even) $r^{-1}$ as follows. First write

$$
\Lambda_{N}^{i}=\stackrel{\circ}{D}_{a} N E_{i}^{a}+q^{-1 / 2} \stackrel{\circ}{D}_{a} q^{1 / 2} N E_{i}^{a}=\stackrel{\circ}{D}_{a} N E_{i}^{a}+(\text { even }) r^{-1}
$$

since $\stackrel{\circ}{D}_{a} q^{1 / 2}=($ odd $) r^{-2}$ and $N=($ odd $) r$. Finally,

$$
\begin{aligned}
\stackrel{\circ}{D}_{a} N E_{i}^{a}-\stackrel{\circ}{\Lambda}_{B}^{i} & =\stackrel{\circ}{D}_{a} N E_{i}^{a}-\stackrel{\circ}{D}_{a} B \stackrel{\circ}{E}_{i}^{a} \\
& =\stackrel{\circ}{D}_{a} N\left(E_{i}^{a}-\stackrel{\circ}{E}_{i}^{a}\right)+\stackrel{\circ}{D}_{a}(N-B) \stackrel{\circ}{E}_{i}^{a} \\
& =(\text { even }) r^{-1} .
\end{aligned}
$$

It then follows that the surface term in 4.30 vanishes and the difference 4.30 is given by the 'pure gauge' Gauss term $-\gamma G\left[\Lambda_{N}-\Lambda_{B}\right]$. Finally, we note that even though we were here mostly interested in the case of boosts, the lapse $N$ above is of the general form (3.24). If $B=0$ the expressions reduce (modulo a pure gauge Gauss term) to the generator of asymptotic time translations $H_{1}[N](3.26)$.

Acknowledgements: I am grateful to Madhavan Varadarajan for his generous guidance over this project, and to Alok Laddha and Sandipan Sengupta for helpful discussions. I would also like to thank AL, MV and Casey Tomlin for their comments on the manuscript.

\section{Appendix A: Assorted results}

1. Eq. 4.10

We starting from an identity given in [1, 11]:

$$
E_{i}^{a} \delta \Gamma_{a}^{i}=\frac{1}{2} \partial_{a}\left(\eta^{a b c} e_{b}^{j} \delta e_{c}^{j}\right)
$$

and rewrite the expression inside the derivative as:

$$
\begin{aligned}
\eta^{a b c} e_{b}^{j} \delta e_{c}^{j} & =e_{b}^{j} \delta\left(\eta^{a b c} e_{c}^{j}\right) \\
& =q^{-1 / 2} e_{b}^{j} \delta\left(q^{1 / 2} \eta^{a b c} e_{c}^{j}\right) \\
& =E_{b}^{j} \delta\left(-\epsilon_{i j k} E_{i}^{a} E_{k}^{b}\right)
\end{aligned}
$$

using the last expression back in (A1) we obtain 4.10.

\section{Integration by parts formulas}

Let $\rho$ be a density one scalar and $X^{a}$ a density one vector field. $\rho$ is dual to the 3 -form $\rho \eta_{a b c}$ and $X^{a}$ dual to the 2 -form $\omega_{a b}:=\eta_{a b c} X^{c}$ so that $X^{a}=\frac{1}{2} \eta^{a b c} \omega_{b c}$.

Stokes theorem for the integral of $\rho=\partial_{a} X^{a}$ over a volume $V$ reads

$$
\int_{V} \partial_{a} X^{a}=\int_{\partial V} d S_{a} X^{a}
$$


In particular, if $\vec{N}$ is a vector field then $\mathcal{L}_{\vec{N}} \rho=\partial_{a}\left(\rho N^{a}\right)$ and one has:

$$
\int_{V} \mathcal{L}_{\vec{N}} \rho=\int_{\partial V} d S_{a} N^{a} \rho
$$

The Lie derivative of $X^{a}$ along a vector field $\vec{N}$ can be written as

$$
\mathcal{L}_{\vec{N}} X^{a}=N^{a} \partial_{b} X^{b}+2 \partial_{b}\left(X^{[a} N^{b]}\right),
$$

where the second term is a total derivative: $2 \partial_{b}\left(X^{[a} N^{b]}\right)=\eta^{a b c} \partial_{b} Y_{c}$ with $Y_{c}=\eta_{c d e} X^{d} N^{e}$. Integrating (A7) over a two-surface $S$ without boundary we obtain the relation:

$$
\oint_{S} d S_{a} \mathcal{L}_{\vec{N}} X^{a}=\oint_{S} d S_{a} N^{a} \partial_{b} X^{b}
$$

\section{Surface term of Eq. (4.7) in terms of $\Gamma_{a}^{i}$ 's}

For a general asymptotic shift, $N^{I}=\alpha^{I}+R^{I}+S^{I}+O\left(r^{-\epsilon}\right)$, Eqns. 4.9 and 4.12 expresses the last term in 4.7 as a pure surface term:

$$
\int_{\Sigma} \Gamma_{a}^{i} \mathcal{L}_{\vec{N}} E_{i}^{a}=\oint d S_{a}\left(N^{a} \Gamma_{b}^{i} E_{i}^{b}+E_{i}^{a} \Lambda_{\vec{N}}^{i}\right)
$$

If we write $\mathcal{L}_{\vec{N}} E_{k}^{a}$ in 4.6 in terms of the derivative $D_{a}$ compatible with $q_{a b}(4.3)$, the second term on the RHS of (A9) is given by:

$$
\oint_{\infty} d S_{a} E_{i}^{a} \Lambda_{\vec{N}}^{i}=\frac{1}{2} \oint_{\infty} d S_{a} \epsilon_{i j k} E_{i}^{a} E_{c}^{j} N^{b} D_{b} E_{k}^{c}-\frac{1}{2} \oint_{\infty} d S_{a} \epsilon_{i j k} E_{i}^{a} E_{c}^{j} E_{k}^{b} D_{b} N^{c} .
$$

The integrand of the second term in A10 can be written as the total derivative $\eta^{a b c} D_{b} N_{c}$ with $N_{c}:=q_{c d} N^{d}$ and hence the integral vanishes. The first term can be cast in terms of the spin connection by use of the formula

$$
\Gamma_{b}^{i}=-\frac{1}{2} \epsilon_{i j k} E_{c}^{j} D_{b} E_{k}^{c}
$$

Doing so one obtains:

$$
\oint_{\infty} d S_{a} E_{i}^{a} \Lambda_{\vec{N}}^{i}=-\oint_{\infty} d S_{a} \Gamma_{b}^{i} N^{b} E_{i}^{a}
$$

which together with the first term in the RHS of (A9) correspond to form of the surface term given in [1].

\section{Appendix B: Eq. 4.16}

Let us denote the 'pure gauge' part of the shift (4.8) by $\vec{\nu}$ so that:

$$
N^{I}=R^{I}+\nu^{I} ; \quad \nu^{I}=S^{I}+O\left(r^{-\epsilon}\right)
$$


Let

$$
\begin{aligned}
\lambda_{\vec{R}}^{i} & :=\frac{1}{2} \epsilon_{i j k}\left(E_{b}^{j} \mathcal{L}_{\vec{R}} E_{k}^{b}-\stackrel{\circ}{E}_{b}^{j} \mathcal{L}_{\vec{R}} \stackrel{\circ}{E}_{k}^{b}\right), \\
\Lambda_{\vec{\nu}}^{i} & :=\frac{1}{2} \epsilon_{i j k} E_{b}^{j} \mathcal{L}_{\vec{\nu}} E_{k}^{b}
\end{aligned}
$$

so that

$$
\lambda_{\vec{N}}^{i}=\lambda_{\vec{R}}^{i}+\Lambda_{\vec{\nu}}^{i}
$$

We now show that the contribution to the surface integral 4.16) from each term in (B4) vanishes. The contribution coming from $\Lambda_{\vec{\nu}}^{i}$ can be written as in A10 with $\vec{N}=\vec{\nu}$. The second term on the RHS of (A10) is again a total derivative whose integral vanishes, whereas the integrand of the first term is now (odd) $r^{-2}+O\left(r^{-2-\epsilon}\right)$ and hence also vanishes.

To study the contribution from $\lambda_{\vec{R}}^{i}$, let us write the triad as

$$
E_{i}^{a}=\stackrel{\circ}{E}_{i}^{a}+g_{i}^{a}+h_{i}^{a},
$$

where $g_{i}^{a}$ represents the (even) $r^{-1}$ term in (3.1) and $h_{i}^{a}$ the remaining $O\left(r^{-1-\epsilon}\right)$ piece. Let

$$
E_{a}^{i}=\stackrel{\circ}{E}_{a}^{i}+g_{a}^{i}+h_{a}^{i}
$$

denote the analgous expansion of the inverse triad so that

$$
\begin{aligned}
g_{a}^{i} & =-\stackrel{\circ}{E}_{b}^{i} \stackrel{\circ}{E}_{a}^{j} g_{j}^{b} \\
h_{a}^{i} & =-\stackrel{\circ}{E}_{b}^{i} \stackrel{\circ}{E}_{a}^{j} h_{j}^{b}+\stackrel{\circ}{E}_{b}^{i} \stackrel{\circ}{E}_{a}^{j} \stackrel{\circ}{E}_{c}^{k} g_{j}^{c} g_{k}^{b}+O\left(r^{-2-\epsilon}\right) .
\end{aligned}
$$

The corresponding expansion for $\lambda_{\vec{R}}$ is:

$$
\lambda_{\vec{R}}^{i}=\frac{1}{2} \epsilon_{i j k}\left(\stackrel{\circ}{E}_{a}^{j} \mathcal{L}_{\vec{R}} g_{k}^{a}+g_{a}^{j} \mathcal{L}_{\vec{R}} \stackrel{\circ}{E}_{k}^{a}+g_{a}^{j} \mathcal{L}_{\vec{R}} g_{k}^{a}+\stackrel{\circ}{E}_{a}^{j} \mathcal{L}_{\vec{R}} h_{k}^{a}+h_{a}^{j} \mathcal{L}_{\vec{R}} \stackrel{\circ}{E}_{k}^{a}\right)+O\left(r^{-2-\epsilon}\right) .
$$

Parity conditions imply that the nontrivial contributions to the integral come from the last two terms in $(\mathrm{B} 9)$ :

$$
\oint d S_{a} E_{i}^{a} \lambda_{\vec{R}}^{i}=\frac{1}{2} \oint d S_{a} \epsilon_{i j k} \stackrel{\circ}{E}_{i}^{a}\left(\stackrel{\circ}{E}_{b}^{j} \mathcal{L}_{\vec{R}} h_{k}^{b}+h_{b}^{j} \mathcal{L}_{\vec{R}} \stackrel{\circ}{E}_{k}^{b}\right)
$$

We now integrate by parts the first term (using Eq. (A8) and $\left.d S_{a} R^{a}=0\right)$ and use (B8) for $h_{b}^{j}$ in the second term (only the first term in (B8) contributes, since the ' $g g$ ' piece is even) to get:

$$
\oint d S_{a} E_{i}^{a} \lambda_{\vec{R}}^{i}=-\frac{1}{2} \oint d S_{a} \epsilon_{i j k}\left(\mathcal{L}_{\vec{R}}\left(\stackrel{\circ}{E}_{i}^{a} \stackrel{\circ}{E}_{b}^{j}\right) h_{k}^{b}+\stackrel{\circ}{E}_{i}^{a} \stackrel{\circ}{E}_{c}^{j} \stackrel{o}{E}_{b}^{l} h_{l}^{c} \mathcal{L}_{\vec{R}} \stackrel{\circ}{E}_{k}^{b}\right)
$$

Using

$$
\begin{aligned}
\mathcal{L}_{\vec{R}}\left(\stackrel{\circ}{E}_{i}^{a} \stackrel{\circ}{E}_{b}^{j}\right) & =-\stackrel{\circ}{E}_{i}^{c} \stackrel{\circ}{E}_{b}^{j} \stackrel{\circ}{D}_{c} R^{a}+\stackrel{\circ}{E}_{i}^{a} \stackrel{\circ}{E}_{c}^{j} \stackrel{\circ}{D}_{b} R^{c} \\
\mathcal{L}_{\vec{R}} \stackrel{\circ}{E}_{k}^{b} & =-\stackrel{\circ}{E}_{k}^{d} \stackrel{\circ}{D}_{d} R^{b}
\end{aligned}
$$

(B11) can be written as (after renaming some indices and using $\stackrel{\circ}{q}_{a b}$ to raise and lower some 
indices):

$$
\oint d S_{a} E_{i}^{a} \lambda_{\vec{R}}^{i}=\frac{1}{2} \oint d S_{a} B^{a b k} \stackrel{\circ}{q} e_{b e} h_{k}^{e}
$$

with

$$
B^{a b k}=\epsilon_{i j k} \stackrel{\circ}{E}_{c}^{i} \stackrel{\circ}{E}_{j}^{b} \stackrel{\circ}{D}^{c} R^{a}-\epsilon_{i j k} \stackrel{\circ}{E}_{i}^{a} \stackrel{\circ}{E}_{c}^{j} \stackrel{\circ}{D}^{b} R^{c}+\epsilon_{i j l} \stackrel{\circ}{E}_{i}^{a} \stackrel{\circ}{E}_{l}^{b} \stackrel{\circ}{E}_{c}^{j} \stackrel{\circ}{E}_{d}^{k} \stackrel{\circ}{D}^{d} R^{c} .
$$

By writing (B15) in cartesian coordinates so that ${\stackrel{\circ}{E_{i}^{a}}}_{i}=\delta_{i}^{a}$, etc. and using that $\stackrel{\circ}{D}^{a} R^{b}=\stackrel{\circ}{\epsilon} a b c \phi_{c}$ for some constant $\phi_{c}$, one finds that $(\mathrm{B} 15)$ identically vanishes:

$$
\begin{aligned}
B^{a b k} & =\epsilon_{c b k} \epsilon_{c a d} \phi_{d}-\epsilon_{a c k} \epsilon_{b c d} \phi_{d}+\epsilon_{a c b} \epsilon_{k c d} \phi_{d} \\
& =\left[\left(\delta_{a b} \delta_{k d}-\delta_{a k} \delta_{b d}\right)-\left(\delta_{a b} \delta_{k d}-\delta_{a d} \delta_{k b}\right)+\left(\delta_{a k} \delta_{b d}-\delta_{a d} \delta_{k b}\right)\right] \phi_{d}=0
\end{aligned}
$$

This concludes the proof of Eq. 4.16.

[1] T. Thiemann, "Generalized boundary conditions for general relativity for the asymptotically flat case in terms of Ashtekar's variables," Class. Quant. Grav. 12, 181 (1995)

[2] R. L. Arnowitt, S. Deser and C. W. Misner, "The Dynamics of general relativity," in "Gravitation: an introduction to current research", Louis Witten ed. (Wiley 1962). [gr-qc/0405109]

[3] T. Regge and C. Teitelboim, "Role of Surface Integrals in the Hamiltonian Formulation of General Relativity," Annals Phys. 88, 286 (1974).

[4] R. Beig and N. ó Murchadha, "The Poincare group as the symmetry group of canonical general relativity," Annals Phys. 174, 463 (1987)

[5] A. Ashtekar, "New Variables for Classical and Quantum Gravity," Phys. Rev. Lett. 57, 2244 (1986)

[6] A. Ashtekar, "New Hamiltonian Formulation of General Relativity," Phys. Rev. D 36, 1587 (1987)

[7] J. F. Barbero G., "Real Ashtekar variables for Lorentzian signature space times," Phys. Rev. D 51, 5507 (1995) [gr-qc/9410014].

[8] G. Immirzi, "Real and complex connections for canonical gravity," Class. Quant. Grav. 14, L177 (1997) [gr-qc/9612030].

[9] G. Date, R. K. Kaul and S. Sengupta, "Topological Interpretation of Barbero-Immirzi Parameter," Phys. Rev. D 79, 044008 (2009) [arXiv:0811.4496 [gr-qc]]

[10] M. Campiglia, M. Varadarajan, "A quantum kinematics for asymptotically flat spacetimes"

[11] T. Thiemann, "Modern canonical quantum general relativity, Cambridge, UK: Cambridge Univ. Pr. (2007) 819 p

[12] A. Ashtekar and J. Lewandowski, "Background independent quantum gravity: A Status report," Class. Quant. Grav. 21, R53 (2004) 